research article

\title{
A phantom to assess the accuracy of tumor delineation using MRSI
}

\author{
Amr A. Heikal, Keith Wachowicz, Steven D. Thomas, Biagio Gino Fallone \\ Department of Medical Physics, Cross Cancer Institute; Departments of Oncology and Physics, \\ University of Alberta, Edmonton, Alberta, Canada
}

\begin{abstract}
Background. Studies have demonstrated that magnetic resonance spectroscopic imaging (MRSI) can detect regions of abnormal activity (tumor) that would not have been covered using conventional imaging and contouring methods. With increased interest in MRSI it is important that its accuracy in tumor delineation be investigated. While some effort has been made to design phantoms to examine the performance of MRSI sequences, most phantoms rely on using traditional glass or acrylic as the phantom building material.

Material and methods. In this work, a gel-based detail phantom has been developed to assess the ability of the spectroscopic imaging sequences to accurately represent the geometry of tumors. The gel-based phantom is used as an alternative to conventional acrylic or glass based phantoms for use with MRSI.

Results. Gel-based phantoms have the advantage of having a magnetic susceptibility close to that of water. In addition, we demonstrate the benefits of having no finite wall thickness separating phantom compartments. The utility of the phantom was illustrated in comparisons between different MRSI sequences of the same nominal resolution as well as different filtering parameters.

Conclusions. Due to their ease of construction and the reduced artifacts, gel phantoms are a reliable tool for assessing the performance of MRSI sequences.
\end{abstract}

Key words: MR spectroscopic imaging; tumor; phantoms; brain; $3 \mathrm{~T}$

\section{Introduction}

The advantages that spectroscopic imaging can offer cancer therapy are significant. By measuring different metabolite levels effectively a means of non-invasive biopsy

Received 23 September 2008

Accepted 1 October 2008

Correspondence to: Amr A. Heikal, Department of Medical Physics, Cross Cancer Institute; Departments of Oncology and Physics, University of Alberta, 11560 University Avenue, Edmonton, Alberta T6G 1Z2, Canada; Phone: +1 780989 4342; Fax: +1-780 432 8615; E-mail: aheikal@phys.ualberta.ca
- magnetic resonance spectroscopic imaging (MRSI) can detect tissue abnormalities that may not yet be visible in conventional MRI. Pirzkall et al. and Pallud et al. have shown that tumor extent as shown by MRSI may differ greatly from the extent shown on conventional MRI scans. ${ }^{1 ; 2}$ Moreover, Walecki et al. have shown that MRSI may help in identifying patients who have a high risk of recurrence. ${ }^{3}$ This has led to an increase in interest in incorporating MRSI into treatment planning by adding a biological target volume in the contouring process. More recently, several methods of 
tumor identification and registration techniques have been used to integrate MRSI results into the radiotherapy process. $4 ; 5$ Such studies have demonstrated that MRSI can detect regions of abnormal activity (tumor) that would not have been covered using conventional imaging and contouring methods. ${ }^{5}$ As such, the delineation of the tumor volume in treatment plans for radiotherapy has changed when MRSI metabolite information was considered.

In the midst of the move to improve the spatial resolution of MRSI, it is important to remember that the ability to properly visualize detailed tumor boundaries is influenced by many imaging and processing parameters, not just the nominal resolution of the scan. Also, with the increased importance of spatial definition of the tumor site for radiotherapy, it is of great importance to have a method of evaluating the accuracy of the boundaries derived from an MRSI sequence under development for use in radiotherapy planning.

While some effort has been made to design phantoms to examine the performance of MRSI sequences; most phantoms have relied on using traditional glass or acrylic as the phantom building material. ${ }^{6,7}$ Detail phantoms utilizing acrylic or glass containers are vulnerable to susceptibility artifacts arising from the interface of the compartment wall material and the solution used to fill the phantom. ${ }^{8}$ Those artifacts are more pronounced in high-detail and irregularly shaped phantoms.

Moreover, detail phantoms rely on a sharply defined boundary between two regions of the phantom. The amount of detail an imaging sequence can reproduce is measured by how accurately it can reproduce that boundary. Having a finite wall containing no metabolites causes a pronounced partial volume artifact in the spectral data. Those artifacts enhance that boundary in a way that is not representa- tive of a human brain. This is demonstrated in the results and discussion section.

In this work, a gel-based detail phantom has been developed to assess the ability of the spectroscopic imaging sequences to accurately represent the detailed geometry of tumors. The gel-based phantom is used as an alternative to conventional acrylic or glass based phantoms for use with MRSI because it avoids susceptibility and compartment wall-related partial volume artifacts. The use of the phantom is demonstrated by comparing the performance of three MRSI sequences.

\section{Material and methods}

\section{Gelatin detail phantom design}

A phantom designed to simulate tumors was constructed using $5 \%$ by weight porcine gel containing clinically relevant concentrations of choline chloride $(3 \mathrm{mM})$ and creatine hydrate $(10 \mathrm{mM}) \cdot{ }^{9}$ A cast acrylic wedge (base: $3.8 \mathrm{~cm}$, height: $9 \mathrm{~cm}$, width: $3.8 \mathrm{~cm}$ ) was inserted in the liquid gel and later removed when the gel hardened. The void left by the wedge was filled with a solution containing elevated levels of choline chloride $(10 \mathrm{mM})$, and the same concentration of creatine as in the background (Figure 1). The higher concentration of choline inside

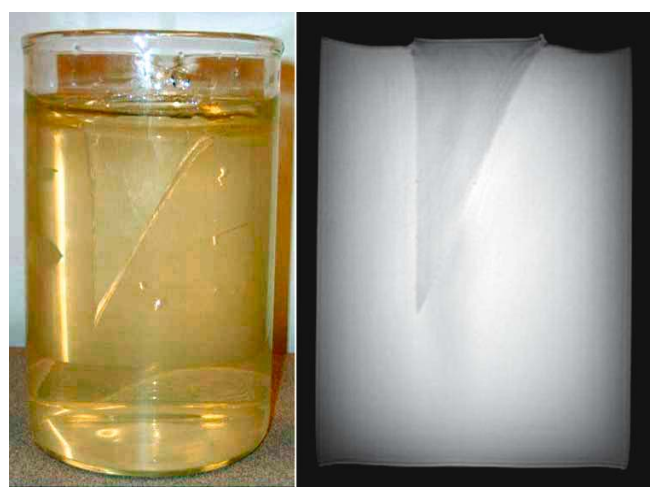

Figure 1. A photograph and a T2 weighted transverse image of the porcine gel detail phantom. 


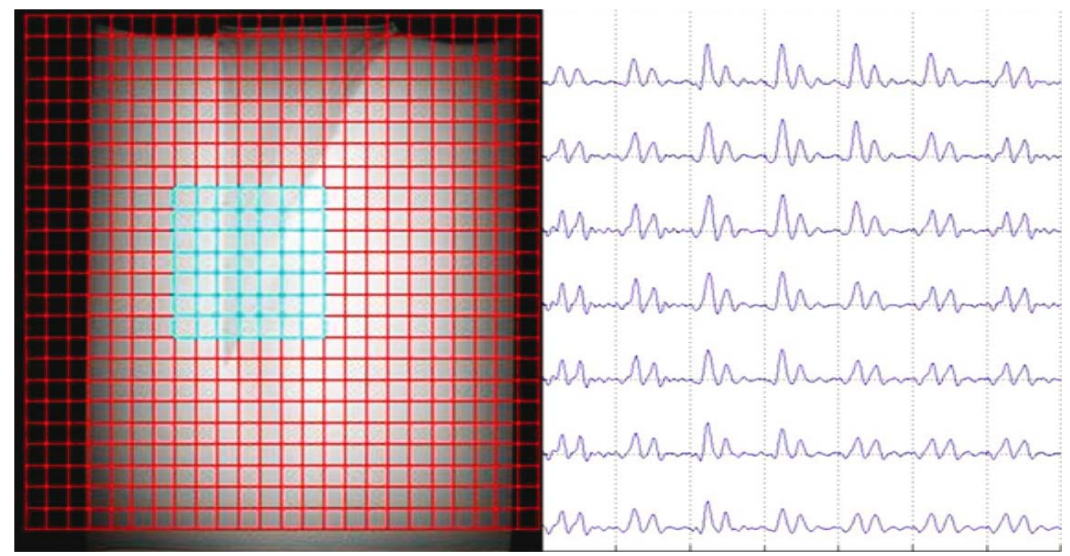

Figure 2. The spectroscopic imaging pixel locations overlaid on the T2 weighted image of the phantom. The spectra on the right correspond to the highlighted pixels. The peaks shown are Choline (left) and Creatine (right). The change in the Choline concentration inside the wedge-shaped compartment is clearly noticeable on the spectra.

the wedge-shaped void was used to simulate the presence of a malignancy.

\section{Comparison of MRSI sequences}

The phantom is designed to assess the ability of the spectroscopic imaging sequences to accurately represent the detailed geometry of tumors. The utility of this phantom was demonstrated by comparing the output of different MRSI sequences with the same nominal resolution. The phantom was scanned with 3 MRSI sequences: 2-D Point Resolved Spectroscopy (PRESS), 2-echo Spin Echo Spectroscopic Imagine (SESI), and 4-echo SESI, all of nominal voxel size $5 \times 5 \times 10 \mathrm{~mm}^{3}$. A $10 \mathrm{~mm}$ thick single-slice T2-weighted image of the phantom was acquired at a position coinciding with the spectroscopic scans (Figure 2). This allowed a proper comparison of the spectroscopic results to the phantom geometry. $\mathrm{Cho} / \mathrm{Cr}$ ratio maps were calculated for each of the spectroscopic scans.

\section{Comparison of $k$-space filters}

The phantom can also be used to optimize filter parameters. Such use is demonstrated by comparing the Cho/Cr maps resulting from the same 2-D PRESS sequence using different $\mathrm{k}$-space filters. Since 2-D cosine k-space filters are routinely used on our system to reduce ringing, it was the type of filter chosen for this demonstration. The phantom was scanned with 2-D PRESS of nominal voxel size $5 \times 5 \times 10 \mathrm{~mm}^{3}$. The data was reconstructed twice; once using no k-space filter and once using a 2-D cosine k-space filter shown in Figure 3. Similar to the previous comparison, a $10 \mathrm{~mm}$ thick single-slice T2-weighted image of the phantom was acquired at a position coinciding with the spectroscopic scans to allow for a proper comparison of the spectroscopic results to the phantom geometry.

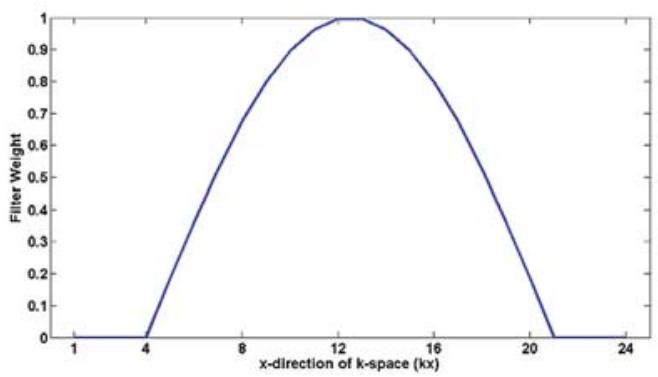

Figure 3. The profile of the 2-D cosine filter in one k-space direction. 


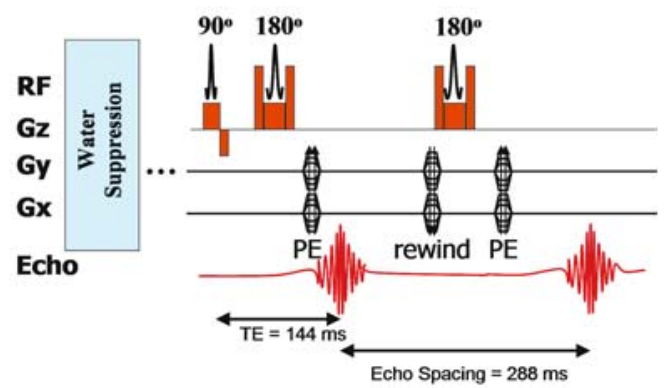

Figure 4. A schematic of a 2-echo SESI MRSI sequence.

\section{Spectroscopic imaging scan parameters}

All scans were performed on a Philips Intera 3 T MRI (Philips Medical Systems, Bothell, WA) unit together with a transmit/ receive birdcage head coil. In this work two types of sequences were used: 2-D PRESS and SESI. The PRESS sequence is a standard sequence used for many in-vivo MRSI studies, especially those interested in high signal-to-noise. ${ }^{10,11}$

SESI offers the choice of acquiring more than one echo per repetition, hence decreasing the overall scan time. ${ }^{11}$ The SESI sequence consists of 2 RF pulses applied on the same plane. The first $90^{\circ}$ pulse excites the slice of interest and then a $180^{\circ}$ refocusing pulse generates an echo at $\mathrm{TE}_{1}$. More echoes can be generated by applying subsequent $180^{\circ}$ refocusing pulses on the same plane each with a different phase encoding (Figure 4). This is analogous to a fast spin echo sequence in imaging.

In the multi-echo SESI sequences the centre of k-space is filled with the first echoes while the edges are filled with data from the later echoes. This produces a T2 weighting artifact in the form of decreased contrast at high spatial resolution compared to 2-D PRESS or single-echo SESI.

For all sequences the TR was set to 1500 $\mathrm{ms}$, and the TE to $144 \mathrm{~ms}$. The number of phase encodes and FOV was set to $24 \times 24$ and $120 \mathrm{~mm}$, respectively, yielding a nominal voxel size of $5 \times 5 \times 10 \mathrm{~mm}^{3}$ for all se- quences. For the SESI sequences the interecho spacing was set to $288 \mathrm{~ms}$.

Prior to running the MRSI scans, second order shimming was established over the volume-of-interest (VOI). ${ }^{12}$ Water suppression was achieved by a mix of chemical shift selective (CHESS) suppression and inversion recovery. ${ }^{13}$; 14 First, a CHESS excitation pulse for water is applied followed by crusher gradients. This yielded a dephased negative water signal that tends to return to equilibrium through $\mathrm{T} 1$ relaxation. The measurement is then acquired at the zero crossing of the water signal in a fashion similar to inversion recovery.

\section{Processing}

The raw data acquired from the MRSI scans were processed on the scanner console using MR Systems Intera (release 1.5.4.3) (Philips Medical Systems, Bothell, WA). First, a cosine filter was applied to the raw k-space data to reduce ringing. The data was then reconstructed to xy-space and adopized by a Lorentzian - Gaussian filter to reduce noise. Zero-order phase, inhomogeneities, and eddy currents were corrected using unsuppressed water measurements collected during the scans. Finally, the frequency domain data was exported to Matlab (The MathWorks, Inc., Natick, MA) where it was further analyzed using inhouse developed software that calculates the area under different metabolite peaks.

\section{Peak fitting}

The in-house developed peak fitting algorithm fitted a sum of Lorentzians to the complex spectra based on seeding values for the chemical shift, estimated peak heights, and full width half maxima (FWHM). ${ }^{15} \mathrm{~A}$ Levenberg-Marquardt algorithm was used to minimize the sum of squares of the difference between the raw spectrum and the 


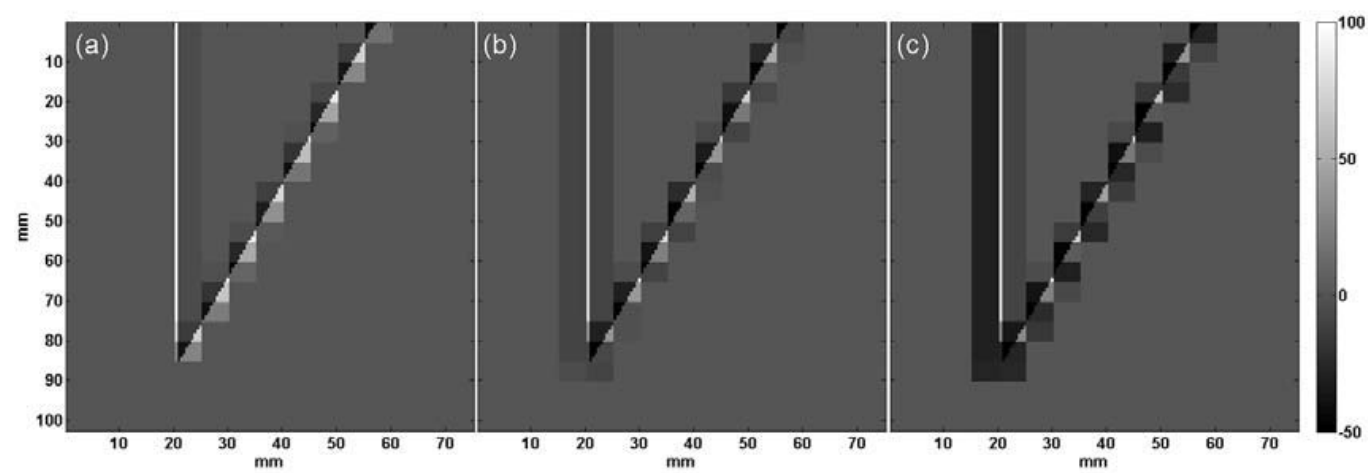

Figure 5. Percentage difference images between simulated $5 \times 5 \mathrm{~mm}^{2}$ pixel images and the phantom geometry using: (a) no compartment wall, (b) $1 \mathrm{~mm}$ wall, (c) $2 \mathrm{~mm}$ wall.

\section{Lorentzians to achieve the best fit. ${ }^{16}$}

The Lorentzian parameters (peak heights, chemical shift, and FWHM) resulting from the peak fitting algorithm were used in the software to analytically calculate the area under the peaks from each spectrum. Since phase was corrected in the processing stage, the real spectrum is assumed to be equal to the absorption spectrum. Hence the area under the peak is calculated as $(\pi / 2 \times \mathrm{FWHM} \times$ peak height). Finally, the software produced metabolite area maps, as well as a choline-to-creatine ratio map.

In-plane interpolation of the metabolite maps has been routinely used in the literature to correlate MRSI with CT and MRI images for treatment planning. ${ }^{17}$ The interpolation smoothes the appearance of the metabolite maps and allows for better comparison with $\mathrm{CT}$ and MRI images. In this work, the metabolite maps were linearly interpolated to $0.5 \mathrm{~mm}$ pixels in-plane.

\section{Results and discussion}

\section{Effects of the phantom wall material}

The rationale behind the choice of the phantom material was to minimize magnetic susceptibility artifacts and partial volume artifacts caused by the presence of compartment walls of finite thickness. While it has been suggested in the literature that the use of water equivalent glass can solve problems of magnetic susceptibility, the problem of wall-related partial volume artifacts would still persist. ${ }^{8}$

The use of porcine gel as the phantom's building material can remedy both these issues. The magnetic susceptibility of the porcine gel is very close to that of water making susceptibility differences negligible. Furthermore, the lack of a physical compartment wall in this design eliminates the issue of its related partial volume artifacts.

In a simple demonstration conducted in Matlab (The MathWorks, Inc., Natick, MA), the two-dimensional geometry of the phantom was modeled using pixel dimensions of $0.5 \mathrm{~mm}$. Derived from a 2-D PRESS scan of a choline filled phantom, the wedge shaped compartment was assigned arbitrary pixel values of 4000 while the pixels outside the wedge were assigned a value of 1500 . A compartment wall was included in the geometric simulation whose thickness was varied between $0 \mathrm{~mm}$ and $2 \mathrm{~mm}$ and was assigned a pixel value of 0 . The pixel values of the $0.5 \mathrm{~mm}$ model were then averaged to $5 \mathrm{~mm}$ pixels, a resolution achievable by MRSI. Figures 5(a), 5(b), and 5(c) show that with a thickness as small as $1 \mathrm{~mm}$, compartment walls can produce visible artifacts 

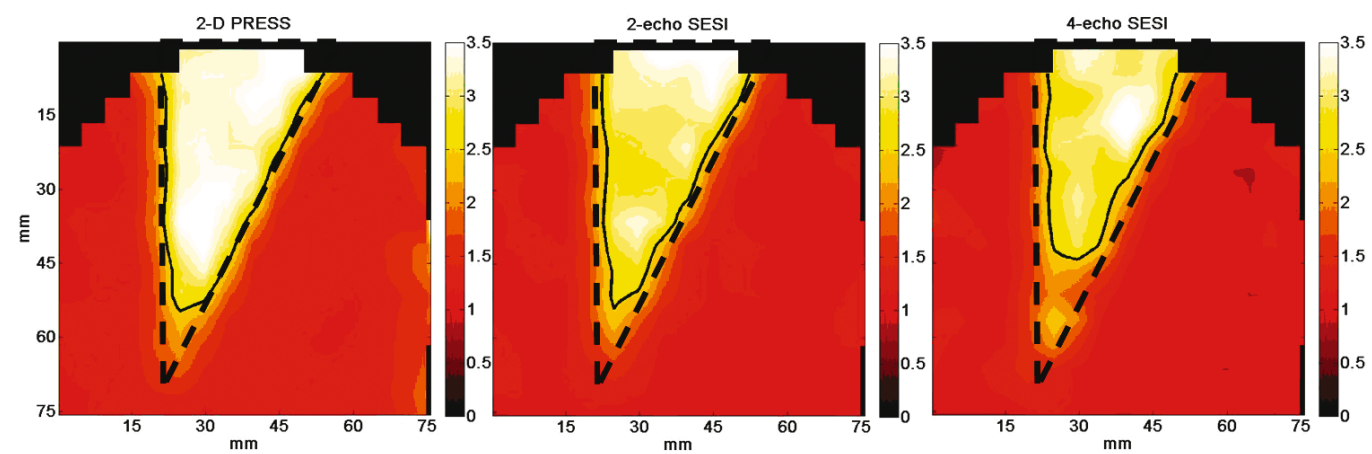

Figure 6. Comparison of the $\mathrm{Cho} / \mathrm{Cr}$ ratio map and the phantom geometry. The scale represents the $\mathrm{Cho} / \mathrm{Cr}$ value. The dashed line represents the phantom geometry and the solid line represents the contour at $\mathrm{Cho} / \mathrm{Cr} \geq 2.5$. The sequences left to right are 2-D PRESS sequence, 2-echo SESI sequence and 4-echo SESI sequence.

that degrade the accuracy and performance of the phantom.

\section{Comparison of MRSI sequences}

Three MRSI sequences of the same nominal resolution were used to illustrate the utility of the gel detail phantom. Figure 6 shows the Cho/Cr ratio maps of a 2-D PRESS, a 2-echo SESI, and a 4-echo SESI sequence compared to phantom geometry.

The tip of the wedge forming the highdetail region of the phantom is intended to show differences in high-detail accuracy between the three sequences. From Figure 6, it is apparent that the 2-D PRESS and the 2echo SESI ratio maps reveal a higher cholineto-creatine ratio extending further into the high detail region of the phantom than in the case of 4-echo SESI. Overall, 2-D PRESS and 2-echo SESI show better conformance to the phantom geometry than 4-echo SESI.

One can quantitatively compare the different sequences by analyzing the deviation between of area of the wedge as shown by the T2 weighted image and the metabolite map. For this purpose the user can choose the suitable tumor contouring criterion for the metabolite maps whether it is a specific metabolite ratio (e.g. Cho/Cr, or Cho/NAA) or z-score (e.g choline-to-NAA index). $5 ; 18 ; 19$ In this demonstration an arbitrary $\mathrm{Cho} / \mathrm{Cr}$ $\geq 2.5$ was chosen as the tumor contouring criterion. The metabolite maps of the three sequences were automatically contoured at that value and the percentage difference of the areas was found to be $13.7 \%, 24.4 \%$, and $38.3 \%$ for 2-D PRESS, 2-echo SESI, and 4echo SESI, respectively. Due to the lack of T2 weighting, 2-D PRESS exhibits the smallest deviation from the phantom geometry. The larger deviations shown by multi-echo SESI can be attributed to T2 losses accumulated in acquiring more than one echo per excitation. Such losses are progressively more evident with the increasing number of echoes per excitation.

\section{Comparison of $k$-space filters}

The ability of MRSI to accurately detect tumor boundaries is sensitive to factors such as k-space sampling and filtering. Like the T2 weighting artifacts discussed earlier, the k-space filter parameters can greatly deteriorate tumor boundary accuracy in MRSI. The phantom was used to reveal differences in tumor delineation resulting from changing $\mathrm{k}$-space filtering. Figure 7 shows the Cho/Cr ratio maps of a 2-D PRESS scan of the phantom reconstructed using unfiltered and 2-D cosine filtered k-space respectively. While the filtered dataset produces fewer noise fluctuations in the ratio map, there 

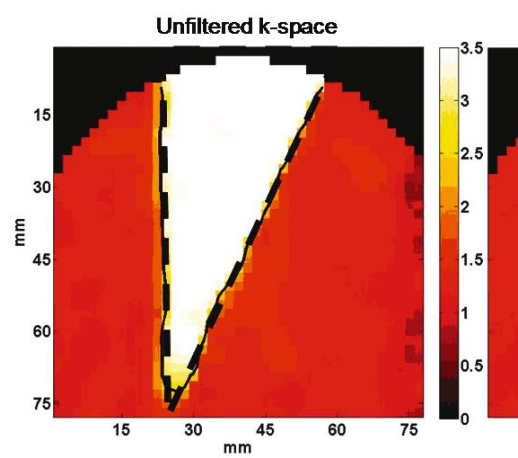

Filtered k-space

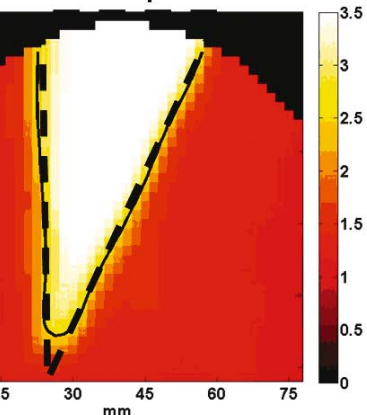

Figure 7. Comparison of the effects of k-space filtering on tumor delineation. The scale represents the $\mathrm{Cho} / \mathrm{Cr}$ value. The dashed line represents the phantom geometry and the solid line represents the contour at $\mathrm{Cho} / \mathrm{Cr} \geq 2.5$. is a much more prominent transition zone visible along the wedge margin. The difference in tumor delineation between the two reconstructions is clear in the lower highdetail region of the wedge shape.

The phantom can be used to evaluate how the detected tumor volume is influenced by different combinations of k-space sampling and filtering during the development phase of a new MRSI sequence. Furthermore, it is useful as a quality assurance tool to ensure the preservation or improvement of the quality of tumor delineation when developing an MRSI sequence or signal processing methods.

\section{Conclusions}

Studies have shown that MRSI has the potential of detecting areas of tumors growth that were otherwise undetectable using conventional imaging. However the ability of MRSI to accurately represent tumor geometry is not always clearly defined by its nominal voxel size, which is influenced by many imaging and processing parameters. It is therefore important to understand and quantify such ability if MRSI is to be used with radiotherapy planning. In this work a detail phantom has been introduced to assist in the process of improving the ability of the spectroscopic imaging sequences to accurately represent the geometry of tumors.
The phantom was developed for assessing the performance of MRSI sequences. Traditionally, MRSI phantoms are susceptible to magnetic susceptibility artifacts and wall-related partial volume artifacts arising from their building material. The use of a porcine gel phantom minimizes the susceptibility artifacts, and the lack of a physical compartmental wall eliminates the associated partial volume artifacts.

The phantom was shown to be successful in demonstrating the differences in tumor boundary definition shown by three MRSI sequences of the same nominal resolution. It was also used to show the differences resulting from applying different k-space filters to the same sequence. There are a number of imaging and filtering parameters that can influence high-resolution contrast of an MRSI sequence. For example, harsh kspace filtering parameters tend to minimize the signal at the edges of k-space. Similarly, increasing the number of echoes acquired per excitation will produce a $\mathrm{T} 2$ weighted k-space, resulting in decreased contrast at high spatial-resolution in the metabolite maps. While a sequence may be designed to achieve a desired nominal resolution, the above mentioned factors can influence the spatial accuracy of tumor boundary delineation to an extent which may not be intuitive to the user. The phantom introduced in this work can be used as a development tool to investigate the effects of those im- 
aging and filtering parameters on the accuracy of tumor delineation before applying the developed sequences clinically. Finally, the reduced artifacts associated with using porcine gel coupled with its relative ease of construction make this kind of phantom a viable option for evaluating the performance of MRSI sequences in both clinical and scientific settings.

\section{Acknowledgements}

Discussion with Dr. Burkhard Maedler from Philips Medical Systems, are appreciated. We acknowledge the financial support of the Canadian Institutes of Health Research, Translational Research Training in Cancer, Canada Foundation for Innovation, and the Alberta Science and Research Investments Program.

\section{References}

1. Pallud J, Devaux B, Nataf F, Roux FX, DaumasDuport C. Spatial delimitation of low grade oligodendrogliomas. Neurochirurgie 2005; 51: 253-9.

2. Pirzkall A, Li X, Oh J, Chang S, Berger MS, Larson DA, et al. 3D MRSI for resected high-grade gliomas before RT: tumor extent according to metabolic activity in relation to MRI. Int J Radiat Oncol Biol Phys 2004; 59: 126-37.

3. Walecki J, Tarasow E, Kubas B, Czemicki Z, Lewko J, Podgorski J, et al. Hydrogen-1 MR spectroscopy of the peritumoral zone in patients with cerebral glioma: assessment of the value of the method. Acad Radiol 2003; 10: 145-53.

4. Chang J, Thakur S, Perera G, Kowalski A, Huang W, Karimi S, et al. Image-fusion of MR spectroscopic images for treatment planning of gliomas. Med Phys 2006; 33: 32-40.

5. McKnight TR, Noworolski SM, Vigneron DB, Nelson SJ. An automated technique for the quantitative assessment of 3D-MRSI data from patients with glioma. J Magn Reson Imaging 2001; 13: 167-77.
6. Hunjan S, Adalsteinsson E, Kim DH, Harsh GR, Boyer AL, Spielman D, et al. Quality assurance of magnetic resonance spectroscopic imaging-derived metabolic data. Int J Radiat Oncol Biol Phys 2003; 57: 1159-73.

7. Woo DC, Kim BS, Jung SL, Park HJ, Rhim HS, Jahng GH, et al. Develpment of a cone-shaped phantom for multi-voxel MR scpectroscopy. J Neurosci Methods 2007; 162: 101-7.

8. Rice JR, Milbrandt RH, Madsen EL, Frank GR, Boote EJ, Blenchinger JC. Anthromorphic ${ }^{1} \mathrm{H}$ MRS head phantom. Med Phys 1998; 25: 1145-56.

9. Barker PB, Hearshen DO, Boska MD. Single-voxel proton MRS of the human brain at 1.5T and 3.0T. Magn Reson Med 2001; 45: 765-9.

10. Drost DJ, Riddle WR, Clarke GD. Proton magnetic resonance spectroscopy in the brain: Report of AAPM Task Group \#9. Med Phys 2002; 29: 2177-97.

11. Nishimura DG. Principles of magnetic resonance imaging: Stanford: Stanford University; 1996.

12. Haacke EM. Magnetic resonance imaging, physics principles and sequence design. New York: John Wiley \& Sons; 1999.

13. Doddrell DM, Galloway G, Brooks W, Filed J, Bulsing J, Irving M, et al. Water signal elimination in vivo, using suppression by mistimed echo and repetitive gradient episodes. J Magn Reson 1986; 70: $176-80$.

14. Hasse A, Frahm J, Hanicke W, Mattaei W. 1H NMR chemical shift selective (CHESS) imaging. Phys Med Biol 1985; 30: 341-4.

15. Mierisova S, Ala-Korpela M. MR spectroscopy quantitation: a review of frequency domain methods. NMR Biomed 2001; 14: 247-59.

16. Moré JJ. The Levenberg-Marquardt algorithm: implementation and theory. In: GA Watson, editor. Lecture notes in mathematics. Heidelberg: Springer Verlag; 1977. p. 105-16.

17. Nelson SJ, Graves E, Pirzkall A, Li X, Chan AA, Vigneron DB, et al. In vivo molecular imaging for planning radiation therapy of gliomas: an application of 1H MRSI. J Magn Reson Imaging 2002; 16: 464-76.

18. Narayana A, Chang J, Thakur S, Huang W, Karimi S, Hou B, et al. Use of MR spectroscopy and functional imaging in the treatment planning of gliomas. Br J Radiol 2007; 80: 347- 54.

19. Jeun SS, Kim MC, Kim BS, Lee JM, Chung ST, Oh CH, et al. Assessment of malignancy in gliomas by $3 \mathrm{~T} 1 \mathrm{H}$ MR spectroscopy. J Clin Imaging 2005; 29: 10-5. 